Pacific

Journal of

Mathematics

NONHOMOGENEOUS BOUNDARY VALUE PROBLEMS

FOR STATIONARY NAVIER-STOKES EQUATIONS IN A MULTIPLY CONNECTED BOUNDED DOMAIN

Hideo KOZONO AND TAKU YANAGISAWA 


\title{
NONHOMOGENEOUS BOUNDARY VALUE PROBLEMS FOR STATIONARY NAVIER-STOKES EQUATIONS IN A MULTIPLY CONNECTED BOUNDED DOMAIN
}

\author{
HideO KOZONO AND TAKU YANAGISAWA
}

\begin{abstract}
We consider the stationary Navier-Stokes equations on a multiply connected bounded domain $\Omega$ in $\mathbb{R}^{n}$ for $n=2,3$ under nonhomogeneous boundary conditions. We present a new sufficient condition for the existence of weak solutions. This condition is a variational estimate described in terms of the harmonic part of solenoidal extensions of the given boundary data; we prove it by using the Helmholtz-Weyl decomposition of vector fields over $\boldsymbol{\Omega}$ satisfying adequate boundary conditions. We also study the validity of Leray's inequality for various assumptions about the symmetry of $\Omega$.
\end{abstract}

\section{Introduction and summary}

We consider the stationary Navier-Stokes equations on a bounded domain $\Omega$ in $\mathbb{R}^{n}$ for $n=2,3$ under nonhomogeneous boundary conditions:

$$
\left\{\begin{aligned}
-\mu \Delta v+(v \cdot \nabla) v+\nabla p & =f & & \text { in } \Omega, \\
\operatorname{div} v & =0 & & \text { in } \Omega, \\
v & =\beta & & \text { on } \partial \Omega .
\end{aligned}\right.
$$

Here $v=v(x)=\left(v_{1}(x), \ldots, v_{n}(x)\right)$ and $p=p(x)$ denote the velocity and pressure at $x=\left(x_{1}, \ldots, x_{n}\right) \in \Omega$, while $f=f(x)$ and $\beta=\beta(x)=\left(\beta_{1}(x), \ldots, \beta_{n}(x)\right)$ denote the given external force defined on $\Omega$ and the given boundary data defined on $\partial \Omega$; the coefficient of viscosity is $\mu>0$. We use standard notation for Laplacian, gradient, divergence, and convective derivative:

$$
\begin{aligned}
\Delta v & =\sum_{j=1}^{n} \frac{\partial^{2} v}{\partial x_{j}{ }^{2}}, & \nabla p & =\left(\frac{\partial p}{\partial x_{1}}, \ldots, \frac{\partial p}{\partial x_{n}}\right), \\
\operatorname{div} v & =\sum_{j=1}^{n} \frac{\partial v_{j}}{\partial x_{j}}, & (v \cdot \nabla) v & =\sum_{j=1}^{n} v_{j} \frac{\partial v}{\partial x_{j}} .
\end{aligned}
$$

MSC2000: 35Q30.

Keywords: stationary Navier-Stokes equations, nonhomogeneous boundary value problems, Helmholtz-Weyl decomposition. 
Throughout, we use conventional notation such as $H^{m}(\Omega), H_{0}^{m}(\Omega), H^{s}(\partial \Omega)$, $W^{s, r}(\Omega)$ for $m \in \mathbb{N}, s>0$ and $1 \leq r \leq \infty$ to denote the usual Sobolev spaces for either scalar or vector functions. We denote by $H_{0, \sigma}^{1}(\Omega)$ the completion of $C_{0, \sigma}^{\infty}(\Omega)$ with respect to the Dirichlet norm $\|\nabla \cdot\|_{L^{2}(\Omega)}$, where $C_{0, \sigma}^{\infty}(\Omega)$ is the set of $u \in C_{0}^{\infty}(\Omega)$ for which $\operatorname{div} u=0$ in $\Omega$; we define $H_{0, \sigma}^{1}(\Omega)^{*}$ to be the dual space of $H_{0, \sigma}^{1}(\Omega)$, and $\langle\cdot, \cdot\rangle$ denotes the duality pairing between $H_{0, \sigma}^{1}(\Omega)^{*}$ and $H_{0, \sigma}^{1}(\Omega)$; the inner product and the norm in $L^{2}(\Omega)$ are denoted by $(\cdot, \cdot)$ and $\|\cdot\|$, respectively.

We also impose throughout the following assumption on $\Omega$.

Assumption 4. (i) The boundary $\partial \Omega$ has connected components $\Gamma_{0}, \Gamma_{1}, \ldots, \Gamma_{L}$, which are $C^{\infty}$ surfaces. The $\Gamma_{1}, \ldots, \Gamma_{L}$ lie inside $\Gamma_{0}$, and $\Gamma_{i} \cap \Gamma_{j}=\varnothing$ for $i \neq j$.

(ii) There exist $C^{\infty}$ surfaces $\Sigma_{1}, \ldots, \Sigma_{N}$ transverse to $\partial \Omega$ such that $\Sigma_{i} \cap \Sigma_{j}=\varnothing$ for $i \neq j$, and such that $\dot{\Omega}=\Omega \backslash \Sigma$ is a simply connected domain, where $\Sigma=\bigcup_{j=1}^{N} \Sigma_{j}$.

In the $n=2$ case, condition (ii) is always fulfilled and the numbers $L$ in (i) and $N$ in (ii) are equal.

As a consequence of the incompressibility condition $\operatorname{div} v=0$ of (1-1), the boundary data $\beta$ is required to satisfy the general flux condition

$$
\sum_{j=0}^{L} \int_{\Gamma_{j}} \beta \cdot v d S=0,
$$

where $v$ is the outward unit normal to $\partial \Omega$.

Suppose that $\beta \in H^{1 / 2}(\partial \Omega)$ and $f \in H_{0, \sigma}^{1}(\Omega)^{*}$. We call $v$ a weak solution of (1-1) if $v \in H^{1}(\Omega)$ satisfies $\operatorname{div} v=0$ in $\Omega, v=\beta$ on $\partial \Omega$, and the integral identity

$$
\mu(\nabla v, \nabla \phi)+((v \cdot \nabla) v, \phi)=\langle f, \phi\rangle
$$

for all $\phi \in H_{0, \sigma}^{1}(\Omega)$. In this paper, we study the existence of weak solutions of (1-1) under the condition (GF).

In his celebrated paper [1933], Leray showed that (1-1) has at least one weak solution under the restricted flux condition

$$
\int_{\Gamma_{j}} \beta \cdot v d S=0 \text { for all } j=0,1, \ldots, L,
$$

which is clearly stronger than the general flux condition (GF). Several fundamental results on the existence and regularity of solutions of (1-1) have since been shown by Hopf [1957], Fujita [1961] and Ladyzhenskaya [1969] under the restricted flux condition (RF). However, it is still unknown whether there exist solutions of (1-1) with boundary data $\beta$ satisfying only the general flux condition (GF). 
One of our main purposes is prove the existence of at least one weak solution under a condition weaker than the restricted flux condition (RF). Our sufficient condition takes the form of a variational estimate (see (1-8) of Theorem 1.3 below) and reflects the topological properties of the domain $\Omega$ explicitly through the space $V_{\text {har }}(\Omega)$ of harmonic vector fields over $\Omega$, defined as the set of $h \in C^{\infty}(\bar{\Omega})$ such that $\operatorname{div} h=0$ and $\operatorname{rot} h=0$ in $\Omega$, and $h \times v=0$ on $\partial \Omega$. The boundary condition appearing in $V_{\text {har }}(\Omega)$ is different from that usually used in the study of NavierStokes equations; see for example [Temam 1979, Theorem 1.5].

In fact, by the Helmholtz-Weyl decomposition of $V_{\text {har }}(\Omega)$ - see Theorem 2.1 we can show a useful criterion on solenoidal extensions of the boundary data $\beta$ :

Proposition 1.1. Let $\Omega$ be a bounded domain in $\mathbb{R}^{n}$ for $n=2,3$ satisfying the assumption ( $\left(\right.$ ). Suppose that the boundary data $\beta \in H^{1 / 2}(\partial \Omega)$ satisfies the general flux condition (GF). Then there exists a solenoidal extension $b \in H^{1}(\Omega)$ of $\beta$ into $\Omega$ such that

$$
\operatorname{div} b=0 \text { in } \Omega \text { and } b=\beta \text { on } \partial \Omega .
$$

Also, any solenoidal extension $b \in H^{1}(\Omega)$ satisfying (1-3) is decomposed as

$$
b=h+\operatorname{rot} w,
$$

where $h \in V_{\mathrm{har}}(\Omega)$ and $w \in X_{\sigma}^{2}(\Omega) \cap H^{2}(\Omega)$, and the following hold:

(I) The vector potential $w$ in (1-4) obeys the estimate

$$
\|w\|_{H^{2}(\Omega)} \leq c\|\beta\|_{H^{1 / 2}(\partial \Omega)},
$$

where $c$ is a constant depending only on $\Omega$,

(II) the harmonic part $h$ in (1-4) is given explicitly as

$$
h=\sum_{\ell=1}^{L} \psi_{\ell} \sum_{j=1}^{L} \alpha_{j \ell} \sum_{k=1}^{L} \alpha_{j k} \int_{\Gamma_{k}} \beta \cdot v d S .
$$

Here $\left\{\psi_{1}, \ldots \psi_{L}\right\}$ is the basis of $V_{\mathrm{har}}(\Omega)$ given below by Theorem 2.1(I) and is related to $q_{j}$ by $\psi_{j}=\nabla q_{j}$ for $j=1, \ldots, L$, while $\left(\alpha_{j k}\right)_{1 \leq j, k \leq L}$ is the $L \times L$ regular matrix defined by

$$
\alpha_{j k}= \begin{cases}\left(1 / \sqrt{\Delta_{j-1} \Delta_{j}}\right) e_{j k} & \text { if } 1 \leq k \leq j, \\ 0 & \text { if } j+1 \leq k \leq L,\end{cases}
$$

where $e_{11}=1$ and $e_{j k}$ with $1 \leq k \leq j$ and $j \geq 2$ denotes the $(j, k)$-cofactor of the matrix

$$
C_{j}=\left(\begin{array}{ccc}
c_{11} & \ldots & c_{1 j} \\
\vdots & \ddots & \vdots \\
c_{j 1} & \ldots & c_{j j}
\end{array}\right) \quad \text { for } 1 \leq j \leq L
$$


with

$$
c_{j k}=\int_{\Gamma_{j}} \frac{\partial q_{k}}{\partial v} d S=\left(\psi_{j}, \psi_{k}\right) \quad \text { for } j, k=1, \ldots, L
$$

and

$$
\Delta_{0}=1 \quad \text { and } \quad \Delta_{j}=\operatorname{det} C_{j} \quad \text { for } 1 \leq j \leq L .
$$

The space $X_{\sigma}^{2}(\Omega)$ appearing in (1-4) is the set of $w \in W^{1,2}(\Omega)$ such that $\operatorname{div} w=0$ in $\Omega$ and $w \cdot v=0$ on $\partial \Omega$.

Remark 1.2. In view of (1-6), the harmonic part $h$ of $b$ depends only on the basis $\left\{\psi_{j}\right\}_{1 \leq j \leq L}$ of $V_{\text {har }}(\Omega)$ and the boundary integrals $\int_{\Gamma_{j}} \beta \cdot v d S$ for $j=1, \ldots, L$. Hence the harmonic part $h$ is independent of the choice of the solenoidal extensions $b$ of the boundary data $\beta$. Also, $h$ can be regarded as the projection of $b$ onto the relative de Rham cohomology $V_{\text {har }}(\Omega)$ of $\Omega$; see [Schwarz 1995, Section 2.6].

With the aid of Proposition 1.1, we can show our main theorem.

Theorem 1.3. For $n=2,3$, suppose $\Omega$ is a bounded domain in $\mathbb{R}^{n}$ satisfying the assumption ( $($ ) $)$. Suppose that the boundary data $\beta \in H^{1 / 2}(\partial \Omega)$ satisfies the general flux condition (GF), and the external force $f$ is in $H_{0, \sigma}^{1}(\Omega)^{*}$. Let $h$ be the harmonic part of the solenoidal extension of $\beta$ into $\Omega$ given by (1-6).

Then, if the estimate

$$
\sup _{z \in \chi(\Omega), \nabla z \neq 0} \frac{(h,(z \cdot \nabla) z)}{\|\nabla z\|^{2}}<\mu
$$

holds, there exists at least one weak solution $v \in H^{1}(\Omega)$ of (1-1). Here

$$
\chi(\Omega)=\left\{z \in H_{0, \sigma}^{1}(\Omega) \mid((z \cdot \nabla) z, \varphi)=0 \text { for all } \varphi \in H_{0, \sigma}^{1}(\Omega)\right\} .
$$

Remark 1.4. (1) The space $\chi(\Omega)$ consists of weak solutions of the stationary Euler equations with Dirichlet boundary condition (see Lemma 2.4). Such a relation between the existence of weak solutions of (1-1) and the space $\chi(\Omega)$ above has been already used tacitly in [Leray 1933], [Amick 1984] and [Kapitanskiǔ and Piletskas 1983] .

(2) By using (1-6), it is not difficult to show that the restricted flux condition (RF) is equivalent to the condition that $h \equiv 0$ in $\Omega$. Hence, the existence of solutions of (1-1) under (RF), already proved in [Leray 1933; Hopf 1957; Fujita 1961; Ladyzhenskaya 1969], can also be derived by applying Theorem 1.3.

As an immediate consequence of Theorem 1.3, we can show that if the harmonic part $h$ of the solenoidal extension of the boundary data $\beta$ is small compared to the viscosity $\mu$, then there exist weak solutions of (1-1).

Corollary 1.5. Let $\Omega, f, \beta$, and $h$ be as in Theorem 1.3. 
(I) $\operatorname{Let} n=3$. If

$$
C_{s}\|h\|_{L^{3}(\Omega)}<\mu,
$$

then there is a weak solution $v \in H^{1}(\Omega)$ of $(1-1)$. Here $C_{s}=3^{-1 / 2} 2^{2 / 3} \pi^{-2 / 3}$ is the best constant of the Sobolev embedding $H^{1}(\Omega) \hookrightarrow L^{6}(\Omega)$.

(II) Let $n=2$ and let $2<p<\infty$. If

$$
C_{q} \Lambda_{1}^{-1 / q}\|h\|_{L^{p}(\Omega)}<\mu
$$

holds for $q=2 p /(p-2)$, then there is a weak solution $v \in H^{1}(\Omega)$ of $(1-1)$. Here $C_{q}$ is the best constant of the Gagliardo-Nirenberg inequality

$$
\|u\|_{L^{q}(\Omega)} \leq C_{q}\|u\|_{L^{2}(\Omega)}^{2 / q}\|\nabla u\|_{L^{2}(\Omega)}^{1-2 / q} \quad \text { for all } u \in H^{1}(\Omega) \text { and } 2<q<\infty,
$$

and $\Lambda_{1}$ is the first eigenvalue of the minus Laplace operator $-\Delta$ under Dirichlet boundary conditions.

Remark 1.6. (1) Galdi [1994, Theorem VIII.4.1] showed in the $n=3$ case that weak solutions of (1-1) exist under a condition somewhat stronger than (1-10). Namely, Galdi assumed that

$$
\sum_{j=1}^{L} k_{j}\left|\int_{\Gamma_{j}} \beta \cdot v d S\right|<v,
$$

where $k_{j}$ for $j=1, \ldots, L$, are certain computable constants depending only on the domain $\Omega$. See also [Borchers and Pileckas 1994, Section 1].

(2) In [Kozono and Yanagisawa 2009b], we proved the result stated in (I) using Hopf's [1957] cut-off function technique and Proposition 1.1. However, the result in (II) for the $n=2$ case seems to be new.

Aside from the corollary above, the variational estimate (1-8) in Theorem 1.3 will give us deeper insight into the existence of weak solutions of (1-1). Indeed, by using the variational estimate (1-8), we can systematically study the validity of Leray's inequality, whose definition we now recall; see [Takeshita 1993]. Suppose that the boundary data $\beta \in H^{1 / 2}(\partial \Omega)$ satisfies the general flux condition (GF). We say Leray's inequality (LI) holds for $\beta$ (and $\Omega$ ) if, for an arbitrary $\epsilon>0$, there is a solenoidal extension $b_{\epsilon}$ of $\beta$ into $\Omega$ such that $\operatorname{div} b_{\varepsilon}=0$ in $\Omega$ and $b_{\varepsilon}=\beta$ on $\partial \Omega$ and such that

$$
\left.\mid(u \cdot \nabla) b_{\varepsilon}, u\right) \mid \leq \varepsilon\|\nabla u\|^{2}
$$

for all $u \in H_{0, \sigma}^{1}(\Omega)$. 
The validity of Leray's inequality leads to an a priori bound of the Dirichlet norm for all possible weak solutions of (1-1), from which the existence of weak solutions of (1-1) immediately follows.

In Section 3, we first observe, by using the Helmholtz-Weyl decomposition again, that if Leray's inequality holds, the numerator $(h,(z \cdot \nabla) z)$ appearing in the variational estimate (1-8) always vanishes for all $z \in \chi(\Omega)$; see Proposition 3.1. In view of this observation, we review the results by Takeshita [1993], Amick [1984] and Fujita [1998], and then give new results on the validity of Leray's inequality under several assumptions about the symmetry of the domain $\Omega$,

This paper is organized as follows. In Section 2, we recall the Helmholtz-Weyl decomposition of $V_{\text {har }}(\Omega)$, which we then use to prove Proposition 1.1. Next, we prove Theorem 1.3 with the aid of Leray and Schauder's fixed point theorem via reduction to absurdity; a key ingredient is Proposition 2.2, a simple observation about the space $\chi(\Omega)$ derived from Proposition 1.1. We then prove Corollary 1.5 by using Theorem 1.3. In Section 3, we study the validity of Leray's inequality. In the appendix, we outline for completeness the proof of the Helmholtz-Weyl decomposition of vector fields over a two-dimensional bounded domain, since we proved it only for the three-dimensional case in [Kozono and Yanagisawa 2009b].

\section{Proof of Proposition 1.1, Theorem 1.3, and Corollary 1.5}

We first give the Helmholtz-Weyl decomposition of the harmonic space $V_{\text {har }}(\Omega)$.

Theorem 2.1. Suppose $\Omega$ is a bounded domain in $\mathbb{R}^{n}$ for $n=2,3$ that satisfies assumption (দ).

(I) The space $V_{\mathrm{har}}(\Omega)$ of harmonic vector fields is L-dimensional. A basis of $V_{\text {har }}(\Omega)$ is the set $\left\{\psi_{1}, \ldots, \psi_{L}\right\}$ such that $\psi_{j}=\nabla q_{j}$ for $j=1, \ldots, L$ where $q_{j}$ solves the Dirichlet boundary value problem of the Laplace equation:

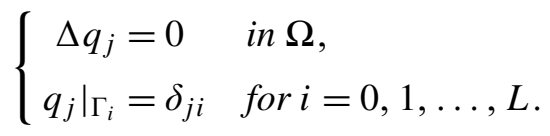

(II) Let $1<r<\infty$. For every $u \in L^{r}(\Omega)$, there exist an $h \in V_{\text {har }}(\Omega)$, a $w \in X_{\sigma}^{r}(\Omega)$ and a $p \in W_{0}^{1, r}(\Omega)$ such that $u$ is decomposed as

$$
u=h+\operatorname{rot} w+\nabla p \quad \text { in } \Omega,
$$

and the triplet $\{h, w, p\}$ in (2-2) satisfies the estimate

$$
\|h\|_{L^{r}(\Omega)}+\|w\|_{W^{1, r}(\Omega)}+\|\nabla p\|_{L^{r}(\Omega)} \leq C\|u\|_{L^{r}(\Omega)},
$$

where $C$ is a constant depending only on $\Omega$ and $r$. This decomposition is unique in that if $u=\tilde{h}+\operatorname{rot} \tilde{w}+\nabla \tilde{p}$ with $\tilde{h} \in V_{\mathrm{har}}(\Omega), \quad \tilde{w} \in X_{\sigma}^{r}(\Omega)$ and $\tilde{p} \in W_{0}^{1, r}(\Omega)$, then $h=\tilde{h}$, rot $w=\operatorname{rot} \tilde{w}$ and $\nabla p=\nabla \tilde{p}$. 
(III) Let $1<r<\infty$ and $s \geq 1$. If $u \in W^{s, r}(\Omega)$ for $1<r<\infty$, then the $w$ and $p$ appearing in (2-2) satisfy

$$
w \in X_{\sigma}^{r}(\Omega) \cap W^{s+1, r}(\Omega) \text { and } \quad p \in W_{0}^{1, r}(\Omega) \cap W^{s+1, r}(\Omega),
$$

and the triplet $\{h, w, p\}$ in (2-2) satisfies

$$
\|h\|_{W^{s, r}(\Omega)}+\|w\|_{W^{s+1, r}(\Omega)}+\|\nabla p\|_{W^{s, r}(\Omega)} \leq C\|u\|_{W^{s, r}(\Omega)},
$$

where $C$ is a constant depending only on $\Omega, s$ and $r$.

The space $X_{\sigma}^{r}(\Omega)$ appearing in statements (II) and (III) is the set of $w \in W^{1, r}(\Omega)$ such that $\operatorname{div} w=0$ in $\Omega$ and $w \cdot v=0$ on $\partial \Omega$. When $n=2$, rot $w$ in (2-2) should be read as $\operatorname{rot} w=\left(\partial w / \partial x_{2},-\partial w / \partial x_{1}\right)$ for a scalar function $w$, and the spaces $V_{\mathrm{har}}(\Omega)$ and $X_{\sigma}^{r}(\Omega)$ should be replaced by $\tilde{V}_{\mathrm{har}}(\Omega)$ and $W_{0}^{1, r}(\Omega)$, respectively, where $\tilde{V}_{\text {har }}(\Omega)$ is the set of $h \in C^{\infty}(\bar{\Omega})$ such that $\operatorname{div} h=0, \quad \operatorname{Rot} h=0$ in $\Omega$ and $h \wedge v=0$ on $\partial \Omega$, with $\operatorname{Rot} h=\partial h_{2} / \partial x_{1}-\partial h_{1} / \partial x_{2}$ and $h \wedge v=h_{2} v_{1}-h_{1} v_{2}$.

Proof of Theorem 2.1. In $n=3$ case, parts (I) and (II) were proved as [Kozono and Yanagisawa 2009c, Theorem 1 part (3) and Theorem 3 part (2)], and see also [Bendali et al. 1985]. To prove part (III), we observe that the scalar potential $p$ and the vector potential $w$ in (2-2) are the solutions of two elliptic boundary value problems

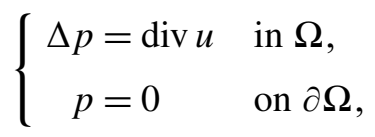

and

$$
\left\{\begin{array}{clrl}
\operatorname{rot} \operatorname{rot} w & =\operatorname{rot} u & & \text { in } \Omega, \\
\operatorname{div} w & =0 & & \text { in } \Omega, \\
\operatorname{rot} w \times v & =u \times v & & \text { on } \partial \Omega, \\
w \cdot v & =0 & & \text { on } \partial \Omega .
\end{array}\right.
$$

In addition, since $-\Delta=$ rot rot - grad div, we find that (2-6) implies that

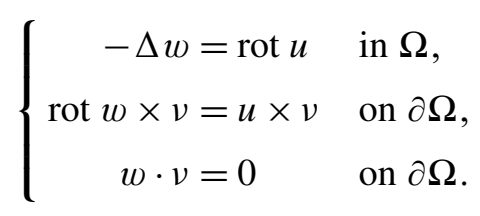

This casts (2-6) into the form of an elliptic boundary value system with complementing boundary conditions in the sense of Agmon, Douglis and Nirenberg; see [Kozono and Yanagisawa 2009c, Lemma 4.3(2)]. Hence, part (III) follows by applying the regularity theorem of [Agmon et al. 1964] to the boundary value 
problems (2-5) and (2-7). The proof of Theorem 2.1 in case when $n=2$ will be separately given in a more general setting in the appendix.

By using Theorem 2.1, we can prove Proposition 1.1.

Proof of Proposition 1.1. Step 1. Since $\beta \in H^{1 / 2}(\partial \Omega)$ satisfies (GF), it is well known that there exists a solenoidal extension $b \in H^{1}(\Omega)$ of $\beta$ into $\Omega$ satisfying (1-3) and

$$
\|b\|_{W^{1,2}(\Omega)} \leq c\|\beta\|_{H^{1 / 2}(\partial \Omega)},
$$

where $c$ is a constant depending only on $\Omega$; see for example [Ladyzhenskaya and Solonnikov 1978].

Step 2. For the solenoidal extension $b$ obtained in the preceding step, we apply Theorem 2.1 with $r=2$ to obtain $b=h+\operatorname{rot} w+\nabla p$, where $w \in X_{\sigma}^{2}(\Omega) \cap H^{2}(\Omega)$, $h \in V_{\text {har }}(\Omega)$, and $p \in W_{0}^{1,2}(\Omega)$. However, since

$$
\Delta p=\operatorname{div} h+\operatorname{div}(\operatorname{rot} w)+\operatorname{div}(\nabla p)=\operatorname{div} b=0 \quad \text { in } \Omega,
$$

and $p=0$ on $\partial \Omega$, we can conclude that $p=0$ in $\Omega$. Therefore, $b=h+\operatorname{rot} w$. The estimate (1-5) follows from the estimates (2-4) with $s=1, r=2$ of Theorem 2.1 and (2-8).

Step 3. By orthogonalization of the basis $\left\{\psi_{j}\right\}_{j=1}^{L}$ of $V_{\text {har }}(\Omega)$ from Theorem 2.1(I), we obtain an orthonormal basis

$$
\varphi_{j}(x)=\sum_{k=1}^{L} \alpha_{j k} \psi_{k}(x) \text { for } j=1, \ldots, L,
$$

where the $\alpha_{j k}$ are the same constants as in (1-7).

By virtue of Theorem 2.1(I), we then see from (2-9) that the harmonic part $h$ of the solenoidal extension $b$ is given as

$$
\begin{aligned}
h & =\sum_{j=1}^{L}\left(b, \varphi_{j}\right) \varphi_{j}=\sum_{j=1}^{L}\left(b, \sum_{k=1}^{L} \alpha_{j k} \psi_{k}\right) \varphi_{j}=\sum_{j, k=1}^{L} \alpha_{j k}\left(b, \nabla q_{k}\right) \varphi_{j} \\
& =-\sum_{j, k=1}^{L} \alpha_{j k}\left(\operatorname{div} b, q_{k}\right) \varphi_{j}+\sum_{j, k=1}^{L} \alpha_{j k} \varphi_{j} \int_{\partial \Omega}(\beta \cdot v) q_{k} d S \\
& =\sum_{j, k=1}^{L} \alpha_{j k} \varphi_{j} \int_{\Gamma_{k}} \beta \cdot v d S .
\end{aligned}
$$


Furthermore, referring to (2-9) again, we have

$$
\begin{aligned}
h & =\sum_{j, k=1}^{L} \alpha_{j k} \varphi_{j} \int_{\Gamma_{k}} \beta \cdot v d S . \\
& =\sum_{j, k=1}^{L} \alpha_{j k} \sum_{\ell=1}^{L} \alpha_{j \ell} \psi_{\ell} \int_{\Gamma_{k}} \beta \cdot v d S \\
& =\sum_{\ell=1}^{L} \psi_{\ell} \sum_{j=1}^{L} \alpha_{j \ell} \sum_{k=1}^{L} \alpha_{j k} \int_{\Gamma_{k}} \beta \cdot v d S .
\end{aligned}
$$

This proves (1-6) and thereby Proposition 1.1.

Proof of Theorem 1.3. The following proposition is crucial for proving Theorem 1.3 and is also part of Section 3's investigation of Leray's inequality.

Proposition 2.2. Suppose that $\beta \in H^{1 / 2}(\partial \Omega)$ satisfies (GF). Let $b \in H^{1}(\Omega)$ be an arbitrary solenoidal extension of $\beta$ into $\Omega$ satisfying (1-3), and let $h$ be the harmonic part of $b$. Then

$$
(b,(z \cdot \nabla) z)=(h,(z \cdot \nabla) z) \quad \text { for all } z \in \chi(\Omega) .
$$

We postpone the proof of Proposition 2.2 to the end of this section. Let $b$ be the solenoidal extension of $\beta$ given by Proposition 1.1. Taking $u=v-b$, we are going to seek a weak solution $u \in H_{0, \sigma}^{1}(\Omega)$ that satisfies

$$
\mu(\nabla u, \nabla \phi)+((b \cdot \nabla) u+(u \cdot \nabla) b+(u \cdot \nabla) u, \phi)=\langle F, \phi\rangle
$$

for all $\phi \in H_{0, \sigma}^{1}(\Omega)$, where $F=\mu \Delta b-(b \cdot \nabla) b+f$. For this purpose, we introduce a parameter $\lambda \in[0,1 / \mu]$ and the equation

$$
\left(\nabla u^{\lambda}, \nabla \phi\right)+\lambda\left((b \cdot \nabla) u^{\lambda}+\left(u^{\lambda} \cdot \nabla\right) b+\left(u^{\lambda} \cdot \nabla\right) u^{\lambda}, \phi\right)=\frac{1}{\mu}\langle F, \phi\rangle,
$$

and put

$$
S(\lambda)=\left\{u^{\lambda} \in H_{0, \sigma}^{1}(\Omega) \mid u^{\lambda} \text { satisfies (2-14) for all } \phi \in H_{0, \sigma}^{1}(\Omega)\right\} .
$$

If we can uniformly bound the Dirichlet norm of all $u^{\lambda} \in S(\lambda)$ as in Lemma 2.3, then (see for example [Ladyzhenskaya 1969] and [Kapitanskiu and Piletskas 1983]) the existence of existence of weak solutions $u \in H_{0, \sigma}^{1}(\Omega)$ satisfying (2-13) for all $\phi \in H_{0, \sigma}^{1}(\Omega)$ will easily follow from Leray and Schauder's fixed point theorem and the homotopy invariance of the degree of the Leray-Schauder mapping.

Lemma 2.3. If the estimate (1-8) in Theorem 1.3 holds, there exists a constant $M$ such that $\left\|\nabla u^{\lambda}\right\| \leq M$ for all $u^{\lambda} \in S(\lambda)$, and for all $\lambda \in[0,1 / \mu]$. 
Proof of Lemma 2.3. We proceed by reduction to absurdity. Suppose that there exist sequences $\left\{u_{j}\right\}_{j=1}^{\infty} \subset H_{0, \sigma}^{1}(\Omega)$ and $\left\{\lambda_{j}\right\}_{j=1}^{\infty} \subset[0,1 / \mu]$ satisfying $\left\|\nabla u_{j}\right\| \rightarrow \infty$ and $\lambda_{j} \rightarrow \lambda_{0} \in[0,1 / \mu]$ as $j \rightarrow \infty$, and

$$
\left(\nabla u_{j}, \nabla \phi\right)+\lambda_{j}\left((b \cdot \nabla) u_{j}+\left(u_{j} \cdot \nabla\right) b+\left(u_{j} \cdot \nabla\right) u_{j}, \phi\right)=\frac{1}{\mu}\langle F, \phi\rangle
$$

for all $\phi \in H_{0, \sigma}^{1}(\Omega)$. Setting $\phi=u_{j}$ in (2-16), we have by integration by parts

$$
\left\|\nabla u_{j}\right\|^{2}+\lambda_{j}\left(\left(u_{j} \cdot \nabla\right) b, u_{j}\right)=\frac{1}{\mu}\left\langle F, u_{j}\right\rangle,
$$

because $b$ satisfies (1-3) and because $u_{j} \in H_{0, \sigma}^{1}(\Omega)$. We then put $w_{j}=u_{j} / N_{j}$ with $N_{j}=\left\|\nabla u_{j}\right\|$ to obtain

$$
1+\lambda_{j}\left(\left(w_{j} \cdot \nabla\right) b, w_{j}\right)=\frac{1}{\mu N_{j}}\left\langle F, w_{j}\right\rangle .
$$

Furthermore, since $\left\|\nabla w_{j}\right\|=1$, we see that the limit $w \in H_{0, \sigma}^{1}(\Omega)$ of $\left\{w_{j}\right\}_{j=1}^{\infty}$ exists in the sense that

(2-18) $\quad \nabla w_{j} \rightarrow \nabla w$ weakly in $L^{2}(\Omega)$ and $\quad w_{j} \rightarrow w$ strongly in $L^{4}(\Omega)$,

as $j \rightarrow \infty$. Therefore, letting $j \rightarrow \infty$ in (2-17), we find by (2-18) that

$$
1+\lambda_{0}((w \cdot \nabla) b, w)=0 .
$$

On the other hand, multiplying both sides of (2-16) by $N_{j}^{-2}$ gives

$\frac{1}{N_{j}}\left(\nabla w_{j}, \nabla \phi\right)+\frac{\lambda_{j}}{N_{j}}\left((b \cdot \nabla) w_{j}+\left(w_{j} \cdot \nabla\right) b, \phi\right)+\lambda_{j}\left(\left(w_{j} \cdot \nabla\right) w_{j}, \phi\right)=\frac{1}{\mu N_{j}^{2}}\langle F, \phi\rangle$,

for all $\phi \in H_{0, \sigma}^{1}(\Omega)$. Letting $j \rightarrow \infty$ in the above, we can also deduce from (2-18) that $\lambda_{0}((w \cdot \nabla) w, \phi)=0$. Since we find by (2-19) that $\lambda_{0} \neq 0$, we have $((w \cdot \nabla) w, \phi)=0$ for all $\phi \in H_{0, \sigma}^{1}(\Omega)$, which implies that $w \in \chi(\Omega)$.

Consequently, if (1-8) in Theorem 1.3 holds, then by Proposition 2.2, we can see from (2-19) that

$$
1=\lambda_{0}(b,(w \cdot \nabla) w)=\lambda_{0}(h,(w \cdot \nabla) w)<\mu \lambda_{0}\|\nabla w\|^{2} \leq\|\nabla w\|^{2},
$$

which contradicts $\|\nabla w\| \leq 1$. This proves Lemma 2.3 and thereby Theorem 1.3.

Proof of Corollary 1.5. In case when $n=3$, by Hölder's inequality and the Sobolev embedding theorem we have

$$
(h,(z \cdot \nabla) z) \leq\|h\|_{L^{3}(\Omega)}\|z\|_{L^{6}(\Omega)}\|\nabla z\|_{L^{2}(\Omega)} \leq C_{s}\|h\|_{L^{3}(\Omega)}\|\nabla z\|_{L^{2}(\Omega)}^{2},
$$

for every $z \in H_{0, \sigma}^{1}(\Omega)$, where $C_{s}$ is the best constant of the Sobolev embedding $H^{1}(\Omega) \hookrightarrow L^{6}(\Omega)$. Therefore, if the condition (1-10) is fulfilled, we see from this inequality that the estimate (1-8) holds. 
In case when $n=2$, we combine the inequalities of Hölder, Poincaré, and Gagliardo and Nirenberg to show that

$$
\begin{aligned}
(h,(z \cdot \nabla) z) & \leq\|h\|_{L^{p}(\Omega)}\|z\|_{L^{q}(\Omega)}\|\nabla z\|_{L^{2}(\Omega)} \\
& \leq C_{q}\|h\|_{L^{p}(\Omega)}\|z\|_{L^{2}(\Omega)}^{2 / q}\|\nabla z\|_{L^{2}(\Omega)}^{2-2 / q} \\
& \leq C_{q} \Lambda_{1}^{-1 / q}\|h\|_{L^{p}(\Omega)}\|\nabla z\|_{L^{2}(\Omega)}^{2},
\end{aligned}
$$

for all $2<p<\infty$ and $1 / q=1 / 2-1 / p$, where $C_{q}$ is the best constant of the Gagliardo-Nirenberg inequality (1-12) and $\Lambda_{1}$ is the first eigenvalue of $-\Delta$ under Dirichlet boundary conditions. Note that $2<q<\infty$ since $2<p<\infty$. Therefore, if (1-11) is satisfied, the estimate (1-8) readily follows from (2-20).

It remains to prove Proposition 2.2. The following lemma regarding the space $\chi(\Omega)$ is a slight modification of the result previously proved by Ladyzhenskaya, Kapitanskiu and Piletskas; see also [Amick 1984].

Lemma 2.4 [Kapitanskiu and Piletskas 1983]. For any $z \in \chi(\Omega)$, there exists a scalar function $q \in W^{1,3 / 2}(\Omega)$ satisfying

$$
(z \cdot \nabla) z+\nabla q=0 \quad \text { in } \Omega .
$$

Furthermore, the trace $\gamma(q) \in W^{1 / 3,3 / 2}(\partial \Omega)$ satisfies

$$
\left.\gamma(q)\right|_{\Gamma_{j}}=c_{j} \quad \text { for } j=0,1, \ldots, L,
$$

where $c_{j}$ is a constant that may depend on $j$.

Proof of Lemma 2.4. Since $z \in H^{1}(\Omega)$, by Hölder's inequality and the Sobolev embedding theorem we see that $(z \cdot \nabla) z \in L^{3 / 2}(\Omega)$. Since $((z \cdot \nabla) z, \varphi)=0$ for all $\varphi \in H_{0, \sigma}^{1}(\Omega)$, by applying the Helmholtz decomposition for $L^{3 / 2}(\Omega)$, we can see that there exists a scalar function $q \in W^{1,3 / 2}(\Omega)$ satisfying $(z \cdot \nabla) z=-\nabla q$ in $\Omega$. That the trace $\gamma(q)$ takes the constant value $c_{j}$ on each boundary component $\Gamma_{j}$ for $j=0,1, \ldots, L$ is proved in [Kapitanskiŭ and Piletskas 1983, Lemma 4].

Proof of Proposition 2.2. Since the boundary data $\beta$ satisfies (GF), we can see by Proposition 1.1 that the solenoidal extension $b \in H^{1}(\Omega)$ of $\beta$ into $\Omega$ decomposes as $b=h+\operatorname{rot} w$, with $h \in V_{\text {har }}(\Omega)$ and $w \in X_{\sigma}^{2}(\Omega) \cap H^{2}(\Omega)$. Therefore, in view of Lemma 2.4, one has by integration by parts

$$
\begin{aligned}
(b,(z \cdot \nabla) z) & =(h+\operatorname{rot} w,(z \cdot \nabla) z) \\
& =(h,(z \cdot \nabla) z)-(\operatorname{rot} w, \nabla q) \\
& =(h,(z \cdot \nabla) z)+\int_{\partial \Omega}(v \times \nabla \gamma(q)) \cdot w d S=(h,(z \cdot \nabla) z)
\end{aligned}
$$

for all $z \in \chi(\Omega)$, because $\left.\gamma(q)\right|_{\Gamma_{j}}=c_{j}$ for $j=0,1, \ldots, L$, and $v \times \nabla$ is a tangential differentiation on the boundary. 


\section{The validity of Leray's inequality}

We begin with a simple but important observation about the relation between the validity of Leray's inequality and the variational estimate (1-8).

Proposition 3.1. Let $\Omega$ be a bounded domain in $\mathbb{R}^{n}$ for $n=2,3$ satisfying the assumption ( $\bigsqcup$ ). Suppose that the boundary data $\beta \in H^{1 / 2}(\partial \Omega)$ satisfies the general flux condition (GF). If Leray's inequality (LI) holds for $\beta$, then

$$
(h,(z \cdot \nabla) z)=0 \quad \text { for all } z \in \chi(\Omega) .
$$

Here $h$, the harmonic part of an arbitrary solenoidal extension $b \in H^{1}(\Omega)$ of $\beta$ into $\Omega$, is as given in (1-6).

Proof of Proposition 3.1. Assume that (LI) holds for $\beta \in H^{1 / 2}(\partial \Omega)$ satisfying (GF). Then, as in the proof of Proposition 1.1, it follows from the HelmholtzWeyl decomposition that any solenoidal extension $b_{\varepsilon}$ of $\beta$ into $\Omega$ decomposes as $b_{\varepsilon}=h+\operatorname{rot} w_{\varepsilon}$, where $h \in V_{\text {har }}(\Omega), w_{\varepsilon} \in X_{\sigma}^{2}(\Omega) \cap H^{2}(\Omega)$. Referring to Remark 1.2, we find that $h$ is independent of $\varepsilon$. Whereas, by Proposition 2.2 we have

$$
\left(b_{\varepsilon},(z \cdot \nabla) z\right)=(h,(z \cdot \nabla) z) \text { for all } z \in \chi(\Omega) .
$$

Therefore, since (LI) holds for $\beta$, we see that for an arbitrary $\varepsilon>0$,

$$
|(h,(z \cdot \nabla) z)|=\left|\left(b_{\varepsilon},(z \cdot \nabla) z\right)\right|=\left|\left((z \cdot \nabla) b_{\varepsilon}, z\right)\right| \leq \varepsilon\|\nabla z\|^{2} .
$$

Since $h$ is independent of $\varepsilon$, we can conclude from (3-2) that

$$
(h,(z \cdot \nabla) z)=0 \quad \text { for all } z \in \chi(\Omega) .
$$

The validity of (3-1) for all $z \in \chi(\Omega)$ implies the estimate (1-8) in Theorem 1.3; we are immediately led from Proposition 3.1 and Theorem 1.3 to this:

Corollary 3.2. Let $\Omega$ and $\beta$ be as in Proposition 3.1. If Leray's inequality (LI) holds for $\beta$, then there exists at least one weak solution $v \in H^{1}(\Omega)$ of (1-1).

Remark 3.3. Let $\beta \in H^{1 / 2}(\partial \Omega)$ satisfy the restricted flux condition (RF) and let $b \in H^{1}(\Omega)$ be an arbitrary solenoidal extension of $\beta$ into $\Omega$. Since (RF) implies that the harmonic part $h$ of $b$ vanishes on $\Omega$, as mentioned in Remark 1.4(2), we can see in a way similar to the proof of Proposition 3.1 that

$$
b=\operatorname{rot} w \quad \text { for some } w \in X_{\sigma}^{2}(\Omega) \cap H^{2}(\Omega) .
$$

Therefore, via Hopf's cut-off function technique [1957], we can conclude that Leray's inequality holds for all $\beta \in H^{1 / 2}(\partial \Omega)$ satisfying (RF).

In view of Remark 3.3, one might ask whether Leray's inequality (LI) holds for all $\beta \in H^{1 / 2}(\partial \Omega)$ satisfying only the general flux condition (GF). According to 
Takeshita [1993], the answer is no. We will give another proof of Takeshita's result by using the following corollary, which is just the contrapositive of Proposition 3.1.

Corollary 3.4. Let $\Omega, \beta$ and $h$ be as in Proposition 3.1. If there exists a vector field $z_{0} \in \chi(\Omega)$ such that

$$
\left(h,\left(z_{0} \cdot \nabla\right) z_{0}\right) \neq 0,
$$

then Leray's inequality (LI) does not hold for $\beta$.

Following [Takeshita 1993], we consider the case when $\Omega$ is an annulus in $\mathbb{R}^{2}$ given by $\Omega=\left\{x \in \mathbb{R}^{2}\left|R_{1}<\right| x \mid<R_{0}\right\}$ with $0<R_{1}<R_{2}$. We put

$$
\Gamma_{0}=\left\{x \in \mathbb{R}^{2}|| x \mid=R_{0}\right\} \quad \text { and } \quad \Gamma_{1}=\left\{x \in \mathbb{R}^{2}|| x \mid=R_{1}\right\} .
$$

Then, from Theorem 2.1(I), one can see that $\operatorname{dim} V_{\text {har }}(\Omega)=1$ and the base of $V_{\text {har }}(\Omega)$ is given by

$$
h=-\frac{x}{2 \pi|x|^{2}} \int_{\Gamma_{1}} \beta \cdot v d S .
$$

Take $z_{0}=f(|x|) e_{\theta}$, with nontrivial function $f(y) \in C_{0}^{\infty}\left(\left(R_{1}, R_{0}\right)\right)$ and unit angular vector $e_{\theta}$. Then it is easy to see that $z_{0}$ is in $H_{0, \sigma}^{1}(\Omega)$ and $\left(z_{0} \cdot \nabla\right) z_{0}=-\nabla q_{0}(|x|)$ with $q_{0}(r)=\int_{R_{1}}^{r} f^{2}(s) / s d s$. Hence, we see that $z_{0} \in \chi(\Omega)$. In addition, we have

$$
\begin{aligned}
\left(h,\left(z_{0} \cdot \nabla\right) z_{0}\right) & =-\left(\left(z_{0} \cdot \nabla\right) h, z_{0}\right) \\
& =\frac{1}{2 \pi} \int_{\Gamma_{1}} \beta \cdot v d S\left(\int_{\Omega} \frac{\left|z_{0}\right|^{2}}{|x|^{2}} d x-\int_{\Omega} \frac{\left(e_{r} \cdot z_{0}\right)^{2}}{|x|} d x\right) \\
& =\int_{\Gamma_{1}} \beta \cdot v d S \int_{R_{1}}^{R_{0}} \frac{f^{2}(r)}{r^{2}} d r,
\end{aligned}
$$

where $e_{r}=x /|x|$. Therefore, if $\int_{\Gamma_{1}} \beta \cdot v d S \neq 0$, then $\left(h,\left(z_{0} \cdot \nabla\right) z_{0}\right) \neq 0$. Combining Corollary 3.4 and Remark 3.3 then gives another proof of Takeshita's result.

Proposition 3.5 [Takeshita 1993]. Let $\Omega$ be an annulus domain in $\mathbb{R}^{2}$ as above. Suppose $\beta \in H^{1 / 2}(\partial \Omega)$ satisfies the general flux condition (GF). Then Leray's inequality (LI) holds for $\beta$ if and only if $\beta$ satisfies the restricted flux condition (RF) as

$$
\int_{\Gamma_{0}} \beta \cdot v d S=\int_{\Gamma_{1}} \beta \cdot v d S=0 .
$$

Remark 3.6. Takeshita [1993, Theorem 2] presented a more general statement: Let $\Omega$ be a bounded domain in $\mathbb{R}^{n}$ with $n \geq 2$ and smooth boundary $\Gamma=\bigcup_{j=1}^{L} \Gamma_{j}$, where the $\Gamma_{j}$ are the connected components of $\Gamma$. Assume that for each such component there exists a diffeomorphism $\Phi_{j}$ of $S^{n-1} \times[0,1]$ into $\bar{\Omega}$ such that $\Phi_{j}\left(S^{n-1} \times\{0\}\right)=\Gamma_{j}$ and $\Phi_{j}\left(S^{n-1} \times\{1\}\right)$ is a sphere contained in $\Omega$. Suppose that $\beta \in H^{1 / 2}(\partial \Omega)$ satisfies (GF). Then (LI) holds for $\beta$ if and only if $\beta$ satisfies (RF). 
Recently, Kobayashi [2009] gave an elementary proof for Takeshita's statement in the two-dimensional case. In [Kozono and Yanagisawa 2009a], we will give a generalization of Takeshita's statement in the three-dimensional case.

From Proposition 3.5 we see that constructive proofs relying on (LI) do not show the existence of weak solutions of (1-1) under the general flux condition (GF) when $\Omega$ is an annulus. This fact, however, does not mean the nonexistence of weak solutions of (1-1).

In fact, Amick [1984] showed the existence of weak solutions of (1-1) under (GF) for a class of symmetric domains $\Omega \subset \mathbb{R}^{2}$, which includes annuli.

Definition 3.7 [Amick 1984]. We say $\Omega \subset \mathbb{R}^{2}$ has type A symmetry if

(i) $\Omega$ is symmetric with respect to the $x_{1}$-axis;

(ii) the boundary $\partial \Omega$ has $L+1$ connected components $\Gamma_{0}, \Gamma_{1}, \ldots, \Gamma_{L}$, which are $C^{\infty}$ surfaces that each intersect the $x_{1}$-axis; the $\Gamma_{1}, \ldots, \Gamma_{L}$ lie inside $\Gamma_{0}$; and $\Gamma_{i} \cap \Gamma_{j}=\phi$ for $i \neq j$.

A vector field $u=\left(u_{1}, u_{2}\right)$ is said to be symmetric (with respect to the $x_{1}$-axis) if $u_{1}$ is an even function of $x_{2}$ and $u_{2}$ is an odd function of $x_{2}$.

Theorem 3.8 [Amick 1984]. Suppose $\Omega$ is a bounded domain in $\mathbb{R}^{2}$ with type $A$ symmetry and smooth boundary. Suppose that the boundary data $\beta^{S} \in H^{1 / 2}(\partial \Omega)$ is symmetric and satisfies the general flux condition (GF), and the external force $f^{S} \in H_{0, \sigma}^{1}(\Omega)^{*}$ is also symmetric. Then there exists at least one symmetric weak solution $v^{S} \in H^{1}(\Omega)$ of (1-1) with $\beta=\beta^{S}$ and $f=f^{S}$.

Amick proved Theorem 3.8 by showing a uniform a priori estimate similar to Lemma 2.3, via reduction of absurdity. The following lemma on the symmetric vector fields of $\chi(\Omega)$ was crucial. Define $\chi^{S}(\Omega)$ to be the space of all symmetric $z^{S} \in H_{0, \sigma}^{1}(\Omega)$ such that $\left(\left(z^{S} \cdot \nabla\right) z^{S}, \varphi\right)=0$ for all $\phi \in H_{0, \sigma}^{1}(\Omega)$.

Lemma 3.9 [Amick 1984]. Suppose $\Omega$ is the domain from Theorem 3.8. Suppose that $z^{S} \in \chi^{S}(\Omega)$ and $q^{S} \in W^{1,3 / 2}(\Omega)$ is the scalar function given in Lemma 2.4 satisfying

$$
\left(z^{S} \cdot \nabla\right) z^{S}+\nabla q^{S}=0 \quad \text { in } \Omega .
$$

Then the trace $\gamma\left(q^{S}\right)$ obeys

$$
\left.\gamma\left(q^{S}\right)\right|_{\Gamma_{j}}=C \quad \text { for } j=0,1, \ldots, L,
$$

where $C$ is a constant independent of $j$.

On the other hand, by retracing the proof of Theorem 1.3, we get the following variant of it in the symmetric case. 
Theorem 3.10. Let $\Omega$ be a bounded domain in $\mathbb{R}^{2}$ that satisfies assumption ( $($ ) and is symmetric with respect to the $x_{1}$-axis. Suppose that $\beta^{S} \in H^{1 / 2}(\partial \Omega)$ is symmetric and satisfies $(\mathrm{GF})$, and the external force $f^{S} \in H_{0, \sigma}^{1}(\Omega)^{*}$ is also symmetric.

Then, if

$$
\sup _{z^{S} \in \chi^{S}(\Omega), \nabla z^{S} \neq 0} \frac{\left(h^{S},\left(z^{S} \cdot \nabla\right) z^{S}\right)}{\left\|\nabla z^{S}\right\|^{2}}<\mu
$$

there exists at least one symmetric weak solution $v^{S} \in H^{1}(\Omega)$ of (1-1) with $\beta=\beta^{S}$ and $f=f^{S}$.

Here $h^{S}$ is the harmonic part of an arbitrary solenoidal extension of $\beta^{S}$ into $\Omega$ defined by (1-6) with $\beta$ replaced by $\beta^{S}$.

Another proof of Theorem 3.8. Let $b^{S} \in H^{1}(\Omega)$ be an arbitrary solenoidal extension of $\beta^{S}$ into $\Omega$ and let $h^{s}$ be its harmonic part given by (1-6). Using (3-4) and Proposition 2.2, one can see by integration by parts that for all $z^{S} \in \chi^{S}(\Omega)$

$$
\begin{aligned}
\left(h^{S},\left(z^{S} \cdot \nabla\right) z^{S}\right) & =\left(b^{S},\left(z^{S} \cdot \nabla\right) z^{S}\right) \\
& =-\left(b^{S}, \nabla q^{S}\right) \\
& =-\int_{\partial \Omega}\left(\beta^{S} \cdot v\right) \gamma\left(q^{S}\right) d S=-C \int_{\partial \Omega} \beta^{S} \cdot v d S=0,
\end{aligned}
$$

because $\beta^{S}$ satisfies (GF). Thus (3-5) holds for all $z^{S} \in \chi^{S}(\Omega)$, and hence we have Theorem 3.8 just by applying Theorem 3.10.

From Takeshita's statement in Remark 3.6, and from the fact that the proof of Theorem 3.10 still relies on reduction to absurdity, one might be tempted to conclude that even when the domain has type A symmetry and all the data is symmetric, it is still hard to give a constructive proof for the existence of weak solutions of (1-1). However, Fujita [1998] succeeded in giving such a proof by showing that Leray's inequality holds if we consider only symmetric test functions $u \in H_{0, \sigma}^{1}(\Omega)$ in $(\mathrm{LI})$.

To make the argument clear, we introduce the symmetric Leray inequality (SLI). Suppose that $\Omega$ is symmetric with respect to the $x_{1}$-axis, and the boundary data $\beta^{S} \in H^{1 / 2}(\partial \Omega)$ satisfies (GF) and is symmetric. We say that the symmetric Leray inequality holds for $\beta^{S}$ (and $\Omega$ ) if, for arbitrary $\varepsilon>0$, there exists a symmetric solenoidal extension $b_{\varepsilon}^{S} \in H^{1}(\Omega)$ of $\beta^{S}$ into $\Omega$ satisfying the inequality

$$
\left.\mid\left(u^{S} \cdot \nabla\right) b_{\varepsilon}^{S}, u^{S}\right) \mid \leq \varepsilon\left\|\nabla u^{S}\right\|^{2}
$$

for all symmetric $u^{S} \in H_{0, \sigma}^{1}(\Omega)$.

Theorem 3.11 [Fujita 1998]. Suppose that $\Omega$ and the boundary data $\beta^{S}$ are as in Theorem 3.8. Then the symmetric Leray inequality (SLI) holds for $\beta^{S}$. 
On the other hand, it is easy to see that the argument that proved Proposition 3.1 yields the following in the symmetric case.

Proposition 3.12. Let $\Omega$ be a bounded domain in $\mathbb{R}^{2}$ that satisfies assumption ( $\emptyset$ ) and that is symmetric with respect to the $x_{1}$-axis. Suppose that the boundary data $\beta^{S} \in H^{1 / 2}(\partial \Omega)$ satisfies the general flux condition (GF) and is symmetric.

Then, if the symmetric Leray inequality (SLI) holds for $\beta^{S}$, we have

$$
\left(h^{S},\left(z^{S} \cdot \nabla\right) z^{S}\right)=0 \quad \text { for all } z^{S} \in \chi^{S}(\Omega) .
$$

Here $h^{S}$ is the harmonic part of an arbitrary solenoidal extension of $\beta^{S}$ into $\Omega$.

Therefore, we can prove the Amick's result in Theorem 3.8 by just combining Theorem 3.11 with Proposition 3.12 and Theorem 3.10.

Definition 3.13. We say $\Omega \subset \mathbb{R}^{2}$ has type B symmetry if

(i) $\Omega$ is symmetric with respect to the $x_{1}$-axis;

(ii) the boundary $\partial \Omega$ has $2 M+1$ connected components $\Gamma_{0}, \Gamma_{1}, \ldots, \Gamma_{2 M}$, which are $C^{\infty}$ surfaces; the components $\Gamma_{1}, \ldots, \Gamma_{2 M}$ lie inside $\Gamma_{0}$ and $\Gamma_{i} \cap \Gamma_{j}=\phi$ for $i \neq j$; and

(iii) the components $\Gamma_{2 j-1}$ and $\Gamma_{2 j}$ for $j=1, \ldots, M$ are symmetric to each other with respect to the $x_{1}$-axis.

Under this symmetry, we will show that the symmetric Leray inequality does not hold for general symmetric boundary data $\beta^{S}$ satisfying (GF), given an additional geometric condition involving the basis of $V_{\mathrm{har}}(\Omega)$ and the space $\chi(\Omega)$.

The following criterion is similar to Corollary 3.4, and is the contrapositive of Proposition 3.12.

Corollary 3.14. Let $\Omega$ and $\beta^{S}$ be as in Proposition 3.12. If there exists a vector field $z_{0}^{S} \in \chi^{S}(\Omega)$ such that

$$
\left(h^{S},\left(z_{0}^{S} \cdot \nabla\right) z_{0}^{S}\right) \neq 0,
$$

then the symmetric Leray inequality (SLI) does not hold for $\beta^{S}$.

Let $\Omega$ be a bounded domain in $\mathbb{R}^{2}$ with type B symmetry, and let $\beta^{S} \in H^{1 / 2}(\partial \Omega)$ satisfy (GF) and be symmetric. We consider first the simplest case that $M=1$, which means that $\partial \Omega$ consists of connected components $\Gamma_{0}, \Gamma_{1}$ and $\Gamma_{2}$; these are $C^{\infty}$ surfaces such that $\Gamma_{1}$ and $\Gamma_{2}$ lie inside of $\Gamma_{0}$, and $\Gamma_{1}$ and $\Gamma_{2}$ are symmetric to each other with respect to the $x_{1}$-axis. We wish to show that there exists a vector field $z_{0}^{S} \in \chi^{S}(\Omega)$ satisfying (3-7) under an additional geometric condition, when the boundary data $\beta^{S}$ does not satisfy (RF). So we first study $V_{\text {har }}(\Omega)$. Let $q_{1}$ be a solution of (2-1) with $j=1$ and $L=2$, so that

$$
\Delta q_{1}=0 \quad \text { in } \Omega,\left.q_{1}\right|_{\Gamma_{j}}=\delta_{1 j} \quad \text { for } j=0,1,2,
$$


and define $q_{2}=q_{2}\left(x_{1}, x_{2}\right)$ by $q_{2}\left(x_{1}, x_{2}\right)=q_{1}\left(x_{1},-x_{2}\right)$. Since $\Gamma_{1}$ and $\Gamma_{2}$ are symmetric to each other with respect to the $x_{1}$-axis, we find that the $q_{2}$ above is a solution of (2-1) with $j=2$ and $L=2$. Hence, we can see that a basis $\left\{\psi_{1}, \psi_{2}\right\}$ of $V_{\text {har }}(\Omega)$ is given by

$$
\psi_{1}(x)=\nabla q_{1}(x), \quad \psi_{2}(x)=\nabla q_{2}(x)=\left(\frac{\partial q_{1}}{\partial x_{1}},-\frac{\partial q_{1}}{\partial x_{2}}\right)\left(x_{1},-x_{2}\right) .
$$

From (1-6), we can see that the harmonic part $h^{S}$ of an arbitrary solenoidal extension of $\beta^{S}$ into $\Omega$ is described as

$$
\begin{aligned}
h^{S}= & \sum_{\ell=1}^{2} \psi_{\ell} \sum_{j=1}^{2} \alpha_{j \ell} \sum_{k=1}^{2} \alpha_{j k} \int_{\Gamma_{k}} \beta^{S} \cdot v d S \\
= & \sum_{\ell=1}^{2} \nabla q_{\ell} \sum_{j=\ell}^{2} \alpha_{j \ell} \sum_{k=1}^{j} \alpha_{j k} \int_{\Gamma_{k}} \beta^{S} \cdot v d S \\
= & \nabla q_{1}\left(\alpha_{11}^{2} \int_{\Gamma_{1}} \beta^{S} \cdot v d S+\alpha_{21}^{2} \int_{\Gamma_{1}} \beta^{S} \cdot v d S+\alpha_{21} \alpha_{22} \int_{\Gamma_{2}} \beta^{S} \cdot v d S\right) \\
& \quad+\nabla q_{2}\left(\alpha_{22} \alpha_{21} \int_{\Gamma_{1}} \beta^{S} \cdot v d S+\alpha_{22}^{2} \int_{\Gamma_{2}} \beta^{S} \cdot v d S\right) \\
= & \left(\left(\alpha_{11}^{2}+\alpha_{22}^{2}+\alpha_{21} \alpha_{22}\right) \nabla q_{1}+\left(\alpha_{22} \alpha_{21}+\alpha_{22}^{2}\right) \nabla q_{2}\right) \int_{\Gamma_{1}} \beta \cdot v d S .
\end{aligned}
$$

In the last equality above, we used the fact that $\int_{\Gamma_{1}} \beta^{S} \cdot v d S=\int_{\Gamma_{2}} \beta^{S} \cdot v d S$, which follows from the symmetry of $\beta^{S}$ and $\Omega$ with respect to the $x_{1}$-axis. Therefore, it holds for all $z^{S} \in \chi^{S}(\Omega)$ that

$$
\begin{aligned}
\left(h^{S},\left(z^{S} \cdot \nabla\right) z^{S}\right)=\left(\left(\alpha_{11}^{2}+\alpha_{21}^{2}+\right.\right. & \left.\alpha_{21} \alpha_{22}\right)\left(\nabla q_{1},\left(z^{S} \cdot \nabla\right) z^{S}\right) \\
& \left.+\left(\alpha_{22} \alpha_{21}+\alpha_{22}^{2}\right)\left(\nabla q_{2},\left(z^{S} \cdot \nabla\right) z^{S}\right)\right) \int_{\Gamma_{1}} \beta \cdot v d S .
\end{aligned}
$$

We put here $k_{i}^{S}=\left(z^{S} \cdot \nabla\right) z_{i}^{S}$ for $i=1,2$. Since $z^{S}$ is symmetric, we find that $k_{1}$ and $k_{2}$ are even and odd, respectively, with respect of $x_{2}$. Hence, by (3-9) and a change of variables, we have

$$
\begin{aligned}
\left(\nabla q_{2},\left(z^{S} \cdot \nabla\right) z^{S}\right) & =\int_{\Omega}\left(\frac{\partial q_{1}}{\partial x_{1}}\left(x_{1},-x_{2}\right) k_{1}^{S}\left(x_{1}, x_{2}\right)-\frac{\partial q_{1}}{\partial x_{2}}\left(x_{1},-x_{2}\right) k_{2}^{S}\left(x_{1}, x_{2}\right)\right) d x \\
& =\int_{\Omega}\left(\frac{\partial q_{1}}{\partial x_{1}}\left(x_{1}, x_{2}\right) k_{1}^{S}\left(x_{1},-x_{2}\right)-\frac{\partial q_{1}}{\partial x_{2}}\left(x_{1}, x_{2}\right) k_{2}^{S}\left(x_{1},-x_{2}\right)\right) d x \\
& =\int_{\Omega}\left(\frac{\partial q_{1}}{\partial x_{1}}\left(x_{1}, x_{2}\right) k_{1}^{S}\left(x_{1}, x_{2}\right)+\frac{\partial q_{1}}{\partial x_{2}}\left(x_{1}, x_{2}\right) k_{2}^{S}\left(x_{1}, x_{2}\right)\right) d x \\
& =\left(\nabla q_{1},\left(z^{S} \cdot \nabla\right) z^{S}\right) .
\end{aligned}
$$


The last two displayed equation then give

$$
\begin{aligned}
\left(h^{S},\left(z^{S} \cdot \nabla\right) z^{S}\right) & =\left(\alpha_{11}^{2}+\alpha_{21}^{2}+2 \alpha_{21} \alpha_{22}+\alpha_{22}^{2}\right)\left(\nabla q_{1},\left(z^{S} \cdot \nabla\right) z^{S}\right) \int_{\Gamma_{1}} \beta^{S} \cdot v d S \\
& =\left(\alpha_{11}^{2}+\left(\alpha_{21}+\alpha_{22}\right)^{2}\right)\left(\nabla q_{1},\left(z^{S} \cdot \nabla\right) z^{S}\right) \int_{\Gamma_{1}} \beta^{S} \cdot v d S .
\end{aligned}
$$

By definition, $\alpha_{11} \neq 0$. Therefore, by Corollary 3.14, the result above gives a theorem:

Theorem 3.15. Let $\Omega$ be a bounded domain in $\mathbb{R}^{2}$ with type B symmetry and $M=1$. Suppose that the boundary data $\beta^{S} \in H^{1 / 2}(\partial \Omega)$ is symmetric and satisfies the general flux condition (GF) as

$$
\int_{\Gamma_{0}} \beta^{S} \cdot v d S+\int_{\Gamma_{1}} \beta^{S} \cdot v d S+\int_{\Gamma_{2}} \beta^{S} \cdot v d S=0
$$

but does not satisfy the restricted flux condition (RF), which means that at least one of these three integrals does not vanish. If there exists a vector field $z_{0}^{S} \in \chi^{S}(\Omega)$ such that

$$
\left(\nabla q_{1},\left(z_{0}^{S} \cdot \nabla\right) z_{0}^{S}\right) \neq 0
$$

then the symmetric Leray inequality (SLI) does not hold for $\beta^{S}$. Here $q_{1}$ is the harmonic function defined by (3-8).

Remark 3.16. By integration by parts, the condition (3-10) is rewritten as

$$
\begin{aligned}
\left(\nabla q_{1},\left(z_{0}^{S} \cdot \nabla\right) z_{0}^{S}\right) & =-\left(\left(z_{0}^{S} \cdot \nabla\right) \nabla q_{1}, z_{0}^{S}\right) \\
& =-\sum_{i, j=1}^{2} \int_{\Omega} \frac{\partial^{2} q_{1}}{\partial x_{i} \partial x_{j}}\left(z_{0}^{S}\right)_{i}\left(z_{0}^{S}\right)_{j} d x=-\int_{\Omega} \operatorname{Hess}\left(q_{1}\right)\left[z_{0}^{S}\right] d x,
\end{aligned}
$$

where $\left(z_{0}^{S}\right)_{j}$ denotes the $j$-th component of $z_{0}^{S}$ and $\operatorname{Hess}\left(q_{1}\right)\left[z_{0}^{S}\right]$ stands for the quadratic form of $z_{0}^{S}$ associated with the Hessian matrix of $q_{1}$. However, because of our lack of our knowledge of the space of $\chi(\Omega)$, it seems difficult to check the validity of (3-10) so far.

We next study bounded domains $\Omega \subset \mathbb{R}^{2}$ with type B symmetry and $M \geq 2$. Suppose $\beta^{S} \in H^{1 / 2}(\partial \Omega)$ is symmetric and satisfies the general flux condition (GF). As before, we let $q_{2 j-1}$ for $j=1, \ldots, M$ solve the boundary value problem

$$
\left\{\begin{aligned}
\Delta q_{2 j-1}=0 & & \text { in } \Omega, \\
\left.q_{2 j-1}\right|_{\Gamma_{i}}=\delta_{2 j-1, i} & & \text { for } i=0,1, \ldots, 2 M,
\end{aligned}\right.
$$

and we define $q_{2 j}$ by $q_{2 j}\left(x_{1}, x_{2}\right)=q_{2 j-1}\left(x_{1},-x_{2}\right)$ for $j=1, \ldots, M$. By the reasoning from the $M=1$ case, we then see that these $q_{2 j}$ solve the boundary 
value problem

$$
\left\{\begin{aligned}
\Delta q_{2 j} & =0 & & \text { in } \Omega, \\
\left.q_{2 j}\right|_{\Gamma_{i}} & =\delta_{2 j, i} & & \text { for } i=0,1, \ldots, 2 M .
\end{aligned}\right.
$$

It follows from Theorem 2.1(I) that the set $\left\{\psi_{1}, \ldots, \psi_{2 M}\right\}$, where

$$
\begin{aligned}
\psi_{2 j-1}(x) & =\nabla q_{2 j-1}(x), \\
\psi_{2 j}(x) & =\nabla q_{2 j}(x)=\left(\frac{\partial q_{2 j-1}}{\partial x_{1}},-\frac{\partial q_{2 j-1}}{\partial x_{2}}\right)\left(x_{1},-x_{2}\right) \quad \text { for } j=1, \ldots, M,
\end{aligned}
$$

is a basis of $V_{\text {har }}(\Omega)$. In the same way as on page 143 , one can also see that

$$
\left(\nabla q_{2 j-1},\left(z^{S} \cdot \nabla\right) z^{S}\right)=\left(\nabla q_{2 j},\left(z^{S} \cdot \nabla\right) z^{S}\right) \quad \text { for } j=1, \ldots, M .
$$

Therefore, by noting the fact that $\int_{\Gamma_{2 j-1}} \beta^{S} \cdot v d S=\int_{\Gamma_{2 j}} \beta^{S} \cdot v d S$ for $j=1, \ldots, M$, we can derive from the above that

$$
\begin{aligned}
\left(h^{S},\left(z^{S} \cdot \nabla\right) z^{S}\right) & \\
= & \sum_{i=1}^{2 M}\left(\nabla q_{i},\left(z^{S} \cdot \nabla\right) z^{S}\right) \sum_{j=1}^{2 M} \alpha_{j i} \sum_{k=1}^{2 M} \alpha_{j k} \int_{\Gamma_{k}} \beta^{S} \cdot v d S \\
= & \sum_{\ell=1}^{M}\left(\nabla q_{2 \ell-1},\left(z^{S} \cdot \nabla\right) z^{S}\right) \sum_{j=1}^{2 M} \alpha_{j, 2 \ell-1} \sum_{k=1}^{2 M} \alpha_{j k} \int_{\Gamma_{k}} \beta^{S} \cdot v d S \\
& +\sum_{\ell=1}^{M}\left(\nabla q_{2 \ell},\left(z^{S} \cdot \nabla\right) z^{S}\right) \sum_{j=1}^{2 M} \alpha_{j, 2 \ell} \sum_{k=1}^{2 M} \alpha_{j k} \int_{\Gamma_{k}} \beta^{S} \cdot v d S \\
= & \sum_{\ell=1}^{M}\left(\nabla q_{2 \ell-1},\left(z^{S} \cdot \nabla\right) z^{S}\right) \sum_{j=1}^{2 M}\left(\alpha_{j, 2 \ell-1}+\alpha_{j, 2 \ell}\right) \sum_{k=1}^{2 M} \alpha_{j k} \int_{\Gamma_{k}} \beta^{S} \cdot v d S \\
= & \sum_{\ell=1}^{M}\left(\nabla q_{2 \ell-1},\left(z^{S} \cdot \nabla\right) z^{S}\right) \\
\quad & \times \sum_{j=1}^{2 M} \sum_{k=1}^{M}\left(\alpha_{j, 2 \ell-1}+\alpha_{j, 2 \ell}\right)\left(\alpha_{j, 2 k-1}+\alpha_{j, 2 k}\right) \int_{\Gamma_{2 k-1}} \beta^{S} \cdot v d S
\end{aligned}
$$

Now for $\ell=1, \ldots, M$, we put

$$
p_{\ell}\left[\beta^{S}\right]=\sum_{j=1}^{2 M} \sum_{k=1}^{M}\left(\alpha_{j, 2 \ell-1}+\alpha_{j, 2 \ell}\right)\left(\alpha_{j, 2 k-1}+\alpha_{j, 2 k}\right) \int_{\Gamma_{2 k-1}} \beta^{S} \cdot v d S
$$


and $q_{\ell}\left[z^{S}\right]=\left(\nabla q_{2 \ell-1},\left(z^{S} \cdot \nabla\right) z^{S}\right)$. Then we can rewrite (3-13) as

$$
\left(h^{S},\left(z^{S} \cdot \nabla\right) z^{S}\right)=\left\langle P\left[\beta^{S}\right], Q\left[z^{S}\right]\right\rangle_{\mathbb{R}^{M}},
$$

where

$$
P\left[\beta^{S}\right]=\left(p_{1}\left[\beta^{S}\right], \ldots, p_{M}\left[\beta^{S}\right]\right) \quad \text { and } \quad Q\left[z^{S}\right]=\left(q_{1}\left[z^{S}\right], \ldots, q_{M}\left[z^{S}\right]\right),
$$

and $\langle\cdot, \cdot\rangle_{\mathbb{R}^{M}}$ denotes the inner product in $\mathbb{R}^{M}$. The next lemma shows that the triviality of $P\left[\beta^{S}\right]$ implies the restricted flux condition (RF).

Lemma 3.17. Let $\Omega$ be a bounded domain in $\mathbb{R}^{2}$ with type $B$ symmetry and $M \geq 2$. Suppose $\beta^{S} \in H^{1 / 2}(\partial \Omega)$ is symmetric and satisfies the general flux condition (GF). Then, $P\left[\beta^{S}\right]=0$ if and only if the restricted flux condition (RF) holds as

$$
\int_{\Gamma_{j}} \beta^{S} \cdot v d S=0 \text { for all } j=0,1, \ldots, 2 M .
$$

Proof of Lemma 3.17. We first observe from (3-14) that

${ }^{t} P\left[\beta^{S}\right]=\left(\sum_{j=1}^{2 M}\left(\alpha_{j, 2 \ell-1}+\alpha_{j, 2 \ell}\right)\left(\alpha_{j, 2 k-1}+\alpha_{j, 2 k}\right) \mid \ell \downarrow 1, \ldots, M, k \rightarrow 1, \ldots, M\right)$

$$
\times\left(\int_{\Gamma_{2 k-1}} \beta^{S} \cdot v d S \mid k \downarrow 1, \ldots, M\right) .
$$

On the other hand, a straightforward calculation yields

$$
\begin{aligned}
& \operatorname{det}\left(\sum_{j=1}^{2 M}\left(\alpha_{j, 2 \ell-1}+\alpha_{j, 2 \ell}\right)\left(\alpha_{j, 2 k-1}+\alpha_{j, 2 k}\right) \mid \ell \downarrow 1, \ldots, M, k \rightarrow 1, \ldots, M\right) \\
& =\sum_{1 \leq j_{1}<\cdots<j_{M} \leq 2 M}\left(\sum_{\sigma=\left(\begin{array}{c}
j_{1}, \ldots, j_{M} \\
k_{1}, \ldots, k_{M}
\end{array}\right)} \operatorname{sgn}(\sigma)\left(\alpha_{k_{1}, 1}+\alpha_{k_{1}, 2}\right) \cdots\left(\alpha_{k_{M}, 2 M-1}+\alpha_{k_{M}, 2 M}\right)\right)^{2},
\end{aligned}
$$

where $\operatorname{sgn}(\sigma)$ is the sign of the permutation $\sigma$. It is easy to see that the right side of this equation contains the term $\left(\alpha_{11} \alpha_{33} \cdots \alpha_{2 M-1,2 M-1}\right)^{2}$, which is positive by the definition of the $\alpha_{j k}$ in Proposition 1.1. Hence, the determinant above is nonzero. Therefore, it follows from (3-16) that $P\left[\beta^{S}\right]=0$ if and only if

$$
\int_{\Gamma_{2 j-1}} \beta^{S} \cdot v d S=0 \quad \text { for all } j=1, \ldots, M \text {. }
$$

It is easy to see that this is equivalent to (RF) since $\beta^{S}$ and $\Omega$ are symmetric with respect to the $x_{1}$-axis and $\beta^{S}$ satisfies (GF). This proves Lemma 3.17.

Accordingly, using Corollary 3.14 again and referring to Lemma 3.17, we have the following theorem. 
Theorem 3.18. Let $\Omega$ be a bounded domain in $\mathbb{R}^{2}$ with type $B$ symmetry and $M \geq 2$. Suppose that the boundary data $\beta^{S} \in H^{1 / 2}(\partial \Omega)$ is symmetric and satisfies the general flux condition (GF) as

$$
\sum_{j=0}^{2 M} \int_{\Gamma_{j}} \beta^{S} \cdot v d S=0,
$$

but does not satisfy the restricted flux condition (RF), which means that at least one of the integrals in the previous expression does not vanish. If there exists a vector fields $z_{0}^{S} \in \chi^{S}(\Omega)$ such that

$$
\left\langle P\left[\beta^{S}\right], Q\left[z_{0}^{S}\right]\right\rangle_{\mathbb{R}^{M}} \neq 0,
$$

then the symmetric Leray inequality (SLI) does not hold for $\beta^{S}$.

Remark 3.19. As can seen from the argument above, it is not difficult to generalize Theorem 3.18 to the case of $\mathbb{R}^{n}$ with $n \geq 3$.

\section{Appendix}

We here outline a proof of the Helmholtz-Weyl decomposition of vector fields over two-dimensional bounded domains; this decomposition is more general than the $n=2$ case of Theorem 2.1. In this appendix, we let

$$
\begin{gathered}
\tilde{X}_{\mathrm{har}}(\Omega)=\left\{h \in C^{\infty}(\bar{\Omega}) \mid \operatorname{div} h=0, \operatorname{Rot} h=0 \text { in } \Omega, h \cdot v=0 \text { on } \partial \Omega\right\}, \\
\tilde{V}_{\mathrm{har}}(\Omega)=\left\{h \in C^{\infty}(\bar{\Omega}) \mid \operatorname{div} h=0, \operatorname{Rot} h=0 \text { in } \Omega, h \wedge v=0 \text { on } \partial \Omega\right\},
\end{gathered}
$$

where Rot $h=\partial h_{2} / \partial x_{1}-\partial h_{1} / \partial x_{2}$ and $h \wedge v=h_{2} v_{1}-h_{1} v_{2}$ for a vector-valued function $h=\left(h_{1}, h_{2}\right)$, and rot $w=\left(\partial w / \partial x_{2},-\partial w / \partial x_{1}\right)$ for a scalar function $w$.

The aim here is to show the following theorem.

Theorem 3.20. Let $\Omega$ be a bounded domain in $\mathbb{R}^{2}$ satisfying the assumption $(\natural)$.

(I) The spaces $\tilde{X}_{\text {har }}(\Omega)$ and $\tilde{V}_{\text {har }}(\Omega)$ are L-dimensional. Furthermore, a basis $\left\{\varphi_{1}, \ldots, \varphi_{L}\right\}$ of $\tilde{X}_{\mathrm{har}}(\Omega)$ and a basis $\left\{\psi_{1}, \ldots, \psi_{L}\right\}$ of $\tilde{V}_{\mathrm{har}}(\Omega)$ are given by

$$
\varphi_{j}=\operatorname{rot} q_{j} \quad \text { and } \quad \psi_{j}=\operatorname{grad} q_{j} \quad \text { for } j=1, \ldots, L,
$$

respectively, where the $q_{j}$ are solutions of the following Dirichlet boundary value problem for the Laplace equation:

$$
\Delta q_{j}=0 \quad \text { in } \Omega \text { and }\left.q_{j}\right|_{\Gamma_{i}}=\delta_{i j} \quad \text { for } i=0,1, \ldots, L .
$$

(II) Let $1<r<\infty$. For every $u \in L^{r}(\Omega)$,

(a) there exists an $h \in \tilde{X}_{\text {har }}(\Omega)$, a $w \in W_{0}^{1, r}(\Omega)$ and $a p \in W^{1, r}(\Omega)$ such that $u=h+\operatorname{rot} w+\nabla p$ in $\Omega$, or 
(b) there exists an $h \in \tilde{V}_{\mathrm{har}}(\Omega)$, a $w \in W^{1, r}(\Omega)$ and $a p \in W_{0}^{1, r}(\Omega)$ such that $u=h+\operatorname{rot} w+\nabla p$ in $\Omega$.

In both cases (a) and (b), the triplet $\{h, w, p\}$ is subject to the estimate

$$
\|h\|_{L^{r}(\Omega)}+\|w\|_{W^{1, r}(\Omega)}+\|\nabla p\|_{L^{r}(\Omega)} \leq C\|u\|_{L^{r}(\Omega)},
$$

where $C$ is a constant depending only on $\Omega$ and $r$. The decompositions in (a) and (b) are unique in the same sense as in Theorem 2.1(II).

(III) Let $1<r<\infty$ and $s \geq 1$. If $u \in W^{s, r}(\Omega)$, then the $w$ and $p$ appearing in the decomposition (a) or (b) gain further regularity such that

$$
w \in W_{0}^{1, r}(\Omega) \cap W^{s+1, r}(\Omega) \text { and } \quad p \in W^{s+1, r}(\Omega) \quad \text { in case (a), }
$$

or

$$
w \in W^{s+1, r}(\Omega) \quad \text { and } \quad p \in W_{0}^{1, r}(\Omega) \cap W^{s+1, r}(\Omega) \text { in case (b). }
$$

In both cases (a) and (b), the triplet $\{h, w, p\}$ is subject to the estimate

$$
\|h\|_{W^{s, r}(\Omega)}+\|w\|_{W^{s+1, r}(\Omega)}+\|\nabla p\|_{W^{s, r}(\Omega)} \leq C\|u\|_{W^{s, r}(\Omega)},
$$

where $C$ is a constant depending only on $\Omega, s$ and $r$.

Proof. The proof proceeds in almost the same way as in [Kozono and Yanagisawa 2009c, Theorem 1]; we call this "our other paper" here. Our other paper studied only the case of three-dimensional bounded domains. Here we will point out only the differences.

Given a vector field $u \in L^{r}(\Omega)$, it is not difficult to see that the scalar functions $p$ and $w$ appearing in case (a) or (b) formally satisfy the following boundary value problems: In case (a),

$$
\begin{aligned}
& \left\{\begin{array}{c}
\Delta p=\operatorname{div} u \quad \text { in } \Omega, \\
\frac{\partial p}{\partial v}=u \cdot v \quad \text { on } \partial \Omega,
\end{array}\right. \\
& \left\{\begin{array}{cl}
\operatorname{Rot} \operatorname{rot} w=\operatorname{Rot} u & \text { in } \Omega, \\
w=0 & \text { on } \partial \Omega,
\end{array}\right.
\end{aligned}
$$

and in case (b)

$$
\begin{aligned}
& \left\{\begin{array}{c}
\Delta p=\operatorname{div} u \quad \text { in } \Omega, \\
p=0 \quad \text { on } \partial \Omega,
\end{array}\right. \\
& \left\{\begin{array}{c}
\operatorname{Rot} \operatorname{rot} w=\operatorname{Rot} u \quad \text { in } \Omega, \\
\frac{\partial w}{\partial v}=u \wedge v \quad \text { on } \partial \Omega .
\end{array}\right.
\end{aligned}
$$


Since the governing boundary value problems (3-20) and (3-22) for $p$ are the same as those in the three-dimensional case, we need only investigate the governing boundary value problems (3-21) and (3-23) for $w$. As in our other paper, we are readily led to weak formulations of solutions of (3-21) and (3-23): In case (a), a scalar function $w \in W_{0}^{1, r}(\Omega)$ is said to be a weak solution of (3-21) if

$$
(\operatorname{rot} w, \operatorname{rot} \varphi)=(u, \operatorname{rot} \varphi)
$$

for any scalar functions $\varphi \in W_{0}^{1, r^{\prime}}(\Omega)$ with $r^{\prime}=r /(r-1)$; in case (b), a scalar function $w \in W^{1, r}(\Omega)$ is a weak solution of (3-23), if

$$
(\operatorname{rot} w, \operatorname{rot} \varphi)=(u, \operatorname{rot} \varphi)
$$

for any scalar functions $\varphi \in W^{1, r^{\prime}}(\Omega)$ with $r^{\prime}=r /(r-1)$.

Then we can easily see that the same procedure from our other paper still works to establish the $L^{r}$-variational inequalities associated with the weak formulations (3-24) and (3-25). By using those $L^{r}$-variational inequalities, we achieve the existence of weak solutions of (3-21) and (3-23). The rest of the proof is word-for-word repetition of the proof in our other paper.

\section{References}

[Agmon et al. 1964] S. Agmon, A. Douglis, and L. Nirenberg, "Estimates near the boundary for solutions of elliptic partial differential equations satisfying general boundary conditions, II", Comm. Pure Appl. Math. 17 (1964), 35-92. MR 28 \#5252 Zbl 0123.28706

[Amick 1984] C. J. Amick, "Existence of solutions to the nonhomogeneous steady Navier-Stokes equations”, Indiana Univ. Math. J. 33:6 (1984), 817-830. MR 86d:35116 Zbl 0563.35059

[Bendali et al. 1985] A. Bendali, J. M. Domínguez, and S. Gallic, "A variational approach for the vector potential formulation of the Stokes and Navier-Stokes problems in three-dimensional domains”, J. Math. Anal. Appl. 107:2 (1985), 537-560. MR 86k:35121 Zbl 0591.35053

[Borchers and Pileckas 1994] W. Borchers and K. Pileckas, "Note on the flux problem for stationary incompressible Navier-Stokes equations in domains with a multiply connected boundary", Acta Appl. Math. 37:1-2 (1994), 21-30. MR 95k:76026 Zbl 0814.76029

[Fujita 1961] H. Fujita, "On the existence and regularity of the steady-state solutions of the NavierStokes theorem”, J. Fac. Sci. Univ. Tokyo Sect. I 9 (1961), 59-102 (1961). MR 24 \#A2152 Zbl 0111.38502

[Fujita 1998] H. Fujita, "On stationary solutions to Navier-Stokes equation in symmetric plane domains under general outflow condition", pp. 16-30 in Navier-Stokes equations: Theory and numerical methods (Varenna, 1997), edited by R. Salvi, Pitman Res. Notes Math. Ser. 388, Longman, Harlow, 1998. MR 2001h:35142

[Galdi 1994] G. P. Galdi, An introduction to the mathematical theory of the Navier-Stokes equations, II: Nonlinear steady problems, Springer Tracts in Natural Philosophy 39, Springer, New York, 1994. MR 95i:35216b Zbl 0949.35005

[Hopf 1957] E. Hopf, "On nonlinear partial differential equations", pp. 1-29 in Lecture Series of the Symposium on Partial Differential Equations (Berkeley, 1955), The University of Kansas, 1957. 
[Kapitanskiı̌ and Piletskas 1983] L. V. Kapitanskiǔ and K. I. Piletskas, "Spaces of solenoidal vector fields and boundary value problems for the Navier-Stokes equations in domains with noncompact boundaries", Trudy Mat. Inst. Steklov. 159:12 (1983), 5-36. In Russian. MR 85a:35074 Zbl 0528.76029

[Kobayashi 2009] T. Kobayashi, “Takeshita’s examples for Leray’s inequality”, preprint, 2009.

[Kozono and Yanagisawa 2009a] H. Kozono and T. Yanagisawa, "Leray's inequality in 3D domains", preprint, 2009.

[Kozono and Yanagisawa 2009b] H. Kozono and T. Yanagisawa, "Leray's problem on the stationary Navier-Stokes equations with inhomogeneous boundary data", Math. Z. 262:1 (2009), 27-39. MR 2491599 Zbl 05547460

[Kozono and Yanagisawa 2009c] H. Kozono and T. Yanagisawa, " $L^{r}$-variational inequality for vector fields and the Helmholtz-Weyl decompositions in bounded domains", preprint, 2009. To appear in Indiana Univ. Math. J.

[Ladyzhenskaya 1969] O. A. Ladyzhenskaya, The mathematical theory of viscous incompressible flow, 2nd ed., Mathematics and its Applications 2, Gordon and Breach Science Publishers, New York, 1969. MR 40 \#7610 Zbl 0184.52603

[Ladyzhenskaya and Solonnikov 1978] O. Ladyzhenskaya and V. Solonnikov, "Some problems of vector analysis and generalized formulation of boundary value problems for the Navier-Stokes equations", J. Soviet Math. 8 (1978), 257-286. Zbl 0388.35061

[Leray 1933] J. Leray, "Etude de diverses équations intégrales non linéaires et de quelques problèmes que pose l'Hydrodyamique", J. Math. Pures Appl. 12 (1933), 1-82. Zbl 0006.16702 JFM 59.0402 .01

[Schwarz 1995] G. Schwarz, Hodge decomposition - a method for solving boundary value problems, Lecture Notes in Mathematics 1607, Springer, Berlin, 1995. MR 96k:58222 Zbl 0828.58002

[Takeshita 1993] A. Takeshita, “A remark on Leray's inequality”, Pacific J. Math. 157:1 (1993), 151-158. MR 93j:35135 Zbl 0801.35108

[Temam 1979] R. Temam, Navier-Stokes equations: Theory and numerical analysis, 2nd ed., Studies in Mathematics and its Applications 2, North-Holland, Amsterdam, 1979. MR 82b:35133 Zbl 0426.35003

Received January 23, 2009. Revised May 16, 2009.

\section{HidEO KOZONO}

MATHEMATICAL InSTITUTE

TOHOKU UNIVERSITY

SENDAI 980-8578

JAPAN

kozono@math.tohoku.ac.jp

TAKU YANAGISAWA

DEPARTMENT OF MATHEMATICS

NARA WOMEN's UNIVERSITY

NARA 630-8506

JAPAN

taku@cc.nara-wu.ac.jp 